Revue de littérature

\title{
Conduites de harcèlement et de cyber-harcèlement chez les adolescents : interrelations et spécificités, place de l'empathie et actions de prise en charge
}

\section{Bullying and cyberbullying among adolescents: Interrelations and specificities, role of empathy and interventions}

\section{Mathys*, S. Claes}

Science politique et criminologie, département de criminologie, faculté de droit, science politique et criminologie, ULiège, quartier Agora, place des Orateurs, 1, bâtiment B 33, 4000 Liège, Belgique

\section{N F O A R T I C L E}

\section{Mots clés :}

Cyber-harcèlement

Adolescence

Empathie

Prévention

\begin{abstract}
R É S U M É
But de l'étude. - Cet article a pour objectif de discuter des conduites de harcèlement et cyber-harcèlement à l'adolescence. Les enjeux cliniques et méthodologiques autour de ces phénomènes seront présentés, avec la volonté de nuancer la compréhension de certains résultats de recherche et réflexes de terrain. Nous proposerons ensuite de définir et circonscrire ces conduites, notamment les interrelations et spécificités de chacune. Dans un troisième temps, nous nous centrerons sur le concept d'empathie et son rôle dans les conduites de harcèlement et cyber-harcèlement concernant l'auteur. Enfin, et en tenant compte des interrelations et distinctions entre harcèlement et cyber-harcèlement et de la place centrale de l'empathie, seront discutées des possibilités d'actions à mener de nature cognitivo-comportementale en vue de prendre en charge ces phénomènes à différents niveaux (micro, méso et macro), ce tant chez l'auteur que chez la victime ou encore plus largement en incluant le groupe de pairs et l'établissement scolaire.
\end{abstract}

(C) 2020 Elsevier Masson SAS. Tous droits réservés.

\section{A B S T R A C T}

Background and objective. - The aim of this study is to examine bullying and cyberbullying among adolescents. Clinical and methodological issues are discussed with the view to nuance some scientific results and common reactions linked to these behaviors. Then, definitions and delimitations of these behaviors are discussed, notably the interrelations and specificities between them. In a next step, the concept of empathy and its implication in bullying and cyberbullying are questioned regarding bullies. Conclusion and implications: some specific and multi-level cognitive-behavioral actions are described to intervene (c) 2020 Elsevier Masson SAS. All rights reserved.

\section{with bullies, victims the peer group and the school environment.}

Keywords:

Cyberbullying

Adolescence

Empathy

Prevention

\section{Entre enjeux cliniques et méthodologiques}

Les conduites de harcèlement à l'adolescence sont reconnues comme un problème de santé publique. En effet, les conséquences peuvent être désastreuses pour chacun des protagonistes. Pour les

\footnotetext{
* Auteur correspondant.

Adresse e-mail : cecile.mathys@uliege.be (C. Mathys).
}

élèves et individus concernés premièrement, tant en termes de santé mentale que de parcours scolaire (phobie scolaire, désengagement et abandon scolaire...), se manifestant chez les victimes (dépression, anxiété, suicide...) et les auteurs (pronostic d'une délinquance persistante, ancrage vers d'autres formes de conduites à risque), et s'accompagnant de troubles internalisés et externalisés accrus pour le statut complexe d'auteur-victime. Également, pour les autres, témoins de situations de harcèlement, actifs ou passifs, qui n'identifient plus l'école comme un environnement sécurisant, 
avec de possibles répercussions sur leurs motivations à apprendre et à fréquenter l'école. Pour les adultes enfin, que ce soient les enseignants, directions d'école, intervenants psycho-sociaux ou encore les parents, souvent impuissants face à une situation qui les dépasse, confrontés à des conduites sans véritables déclencheurs et pour lesquelles la réponse individuelle n'est pas suffisante.

Aujourd'hui, ce phénomène est encore amplifié par l'utilisation des nouvelles technologies d'information et de communication. Une série de questions se pose donc : qui est concerné par le phénomène ? Y a-t-il un profil et des caractéristiques que l'on retrouve davantage associés chez une potentielle victime ou un probable auteur de ce type de conduites ? Est-ce que le harcèlement et le cyber-harcèlement partagent les mêmes mécanismes et recouvrent un seul et même concept ? Ces questionnements, anodins, sont pourtant cruciaux puisqu'ils conditionnent la façon dont ces conduites vont être appréhendées, voire identifiées, pour ensuite être régulées.

En effet, en matière d'actions à mener, les facteurs reconnus comme participant au phénomène de harcèlement au sens large, c'est-à-dire existant avant son apparition, s'avèrent déterminants dans la conception et la mise en place de la prise en charge [1]. Par exemple, si le retrait social est reconnu comme un facteur de risque, offrir des interventions qui diminueraient le retrait social des élèves et/ou axeraient sur le collaboratif, permettrait de prévenir les victimisations de harcèlement traditionnel. Ce facteur n'étant pas relié au cyber-harcèlement, agir à ce niveau n'offrirait que peu de bénéfices, si ce n'est celui, humain, de réagir. A contrario, si les fondements sont communs, une réaction singulière, agir sur tel facteur, pourrait être efficace et permettrait de participer à prévenir l'apparition et le développement de conduites de harcèlement et de cyber-harcèlement. En tant que professionnel, la tentation serait grande de pouvoir utiliser " une même formule " pour gérer un ensemble de comportements problématiques. Toutefois, estce pertinent scientifiquement ? Des enjeux méthodologiques et conceptuels, concernant la définition même du phénomène, sont donc présents.

Cet article a dès lors pour objectif d'éclairer la compréhension des conduites de harcèlement et de cyber-harcèlement à l'adolescence en abordant des éléments de contextualisation. Premièrement, nous discuterons de la définition de ces phénomènes et des liens et enjeux les réunissant. Ensuite, nous nous centrerons sur une composante essentielle qu'est l'empathie, avec de nouveau le souhait de définir et comprendre clairement ce qu'est l'empathie et son rôle dans les conduites de harcèlement et de cyber-harcèlement, notamment concernant l'auteur. Enfin, et en tenant compte des interrelations et distinctions entre harcèlement et cyber-harcèlement ainsi que de la place centrale de l'empathie, seront discutées de possibilités d'actions de nature cognitivo-comportementale à mener en vue d'intervenir sur ces phénomènes à différents niveaux (micro, méso et macro) et selon les protagonistes impliqués.

\section{Prévalence et définitions}

Le harcèlement scolaire serait perçu comme la forme de violence la plus répandue au sein des cours de récréation. La plupart des études internationales auto-révélées [2] indiquent une prévalence de ce phénomène d'environ $35 \%$ au sein de la population adolescente scolarisée. Plus précisément, une étude américaine s'intéressant à l'évolution du harcèlement sur 10 ans (2005-2014) démontre une baisse des taux de victimisation de $28,8 \%$ à $13,4 \%$ et de perpétration de $21,3 \%$ à 7 \% [3]. Les études réalisées dans les pays francophones ${ }^{1}$ obtiennent des taux de prévalence concernant les victimes de harcèlement chez des adolescents âgés de 11 à 15 ans allant de $11,5 \%$ à $20 \%$ [4]. Tandis qu'une étude espagnole observe chez des élèves de 9 à 13 ans des taux de prévalence de $23,9 \%$ pour le statut de victime-auteur et de $28,9 \%$ pour le statut de témoin d'un épisode d'harcèlement durant l'année précédant l'enquête [5]. Il est ainsi observé que les taux d'implication semblent très variables d'un pays à l'autre selon la définition choisie pour étudier le harcèlement et donc les conduites ciblées, ainsi que les protagonistes impliqués (auteur, victime, double statut, témoins) ou encore la période développementale considérée.

Il en est de même concernant le phénomène de cyberharcèlement. Une revue internationale de la littérature fait état de prévalences de victimisations liées au cyber-harcèlement se situant entre $6,8 \%$ et $35,4 \%$ au sein d'un échantillon âgés de 10 à 17 ans [6]. Une étude comparative européenne observe des tendances comparables ( $21,4 \%$ en moyenne) pour un échantillon de 14-18 ans, avec une mesure de la cyber-victimisation évaluée sur les 12 derniers mois [7]. Dans une étude récente [8] menée auprès de 4390 adolescents italiens âgés de 13 à 18 ans, 36,5\% de garçons et $22,9 \%$ de filles indiquent être auteurs de cyber-harcèlement contre 30,1 \% de garçons et 32,5 \% de filles qui révèlent être victimes de cyber-harcèlement sur les six derniers mois. Enfin, dans une étude américaine s'intéressant à la cyber-victimisation auprès d'un échantillon plus âgé (244 étudiants de 18-25 ans), 55 \% d'entre eux affirment avoir témoin d'actes de cyber-harcèlement au moins une fois durant l'année écoulée [9].

Pour mieux comprendre ces variations de prévalences, il convient de s'intéresser à la définition même des phénomènes de harcèlement et de cyber-harcèlement. Olweus [10] spécifie le harcèlement en lui assignant 3 critères : un comportement agressif ou blessant de manière intentionnelle qui se répète à maintes reprises et qui est caractérisé par un déséquilibre de pouvoir entre les protagonistes. Cette définition est généralement utilisée comme référence par la littérature scientifique. Cependant, certains auteurs y ajoutent d'autres critères. Ainsi, Smith et al. [11] insistent pour que le comportement se produise sans qu'il n'y ait de provocation de la part de la victime. D'autres observent que l'intentionnalité de l'acte est vérifiée mais pas forcément pour nuire intentionnellement à autrui [12]. Enfin, la notion de déséquilibre de pouvoir qui est vécue comme telle par la victime, indépendamment de caractéristiques physiques observées, se traduirait par une impossibilité de mettre fin à la situation de harcèlement [13]. De manière générale, la notion de répétition des actes est un point essentiel de la définition. Le harcèlement se manifeste sous des formes et espaces variés, les plus courants étant physiques, verbaux, relationnels ou virtuels [13]. Nous percevons déjà ici toute la complexité de mesurer rigoureusement le phénomène selon que la question posée au sein d'une enquête auto-révélée intégrerait une idée d'intentionnalité sous forme de nuisance ( " je me suis délibérément moqué de quelqu'un pour l'embêter »), qui ici participerait à sous-estimer largement le phénomène, en identifiant un profil d'auteur, plutôt rare, à prédisposition psychopathique ; ou encore questionnerait la conduite sans y associer le critère nécessaire de répétition. À cet égard, il s'agit d'examiner en détail le type de répétitions. Pour être le plus précis possible, la prévalence du comportement et sa fréquence devraient être intégrées à différents moments dans le temps (" estce déjà arrivé ", si oui, combien de fois ? ", "à quel moment dans le temps »), ce afin d'identifier les formes chroniques et graves de harcèlement [13]. Enfin, ces définitions, bien que semblant faire consensus, ne mettent pas suffisamment en évidence la position du groupe de pairs, acteur clé dans les situations de harcèlement,

\footnotetext{
${ }^{1}$ France, Suisse, Canada, Belgique.
} 
ce qui conditionne, selon nous, une action ciblée sur l'auteur et la victime, ce que la littérature scientifique dénonce par ailleurs [14].

Concernant le cyber-harcèlement, Sourander et al. [15] le définissent comme " un acte intentionnel et agressif mené par un groupe ou un individu utilisant des moyens de contact électroniques, de manière répétée dans le temps à l'encontre d'une victime qui ne peut pas facilement se défendre "[p.720]. L'espace dans lequel s'exprime la conduite de cyber-harcèlement est ici central et intègre les domaines du numérique et des nouvelles technologies. Cette émergence des nouveaux moyens de communication s'accompagne donc d'une forme plus spécifique de harcèlement. Dans le cas du cyber-harcèlement, la pluralité des espaces est à intégrer dans la définition et l'investigation du phénomène, au risque, ici aussi, de sous-estimer le phénomène si celui-ci se réduit à " ce que je reçois sur Facebook ».

Aussi, nous rejoignons Rivers et al. [16] qui suggèrent que la méthode selon laquelle les chercheurs mesurent les conduites de harcèlement et de cyber-harcèlement doit être prise en compte dans l'analyse des résultats et la compréhension des chiffres communiqués ; invitant à une nécessaire posture de prudence voire de réfutabilité.

\section{Liens entre harcèlement et cyber-harcèlement : un ou deux phénomènes ?}

Le cyber-harcèlement entre pairs, historiquement considéré comme une suite au harcèlement classique/scolaire dans un espace différencié [13], est actuellement remis en question. En effet, un deuxième courant de pensée issu des 20 dernières années permet de réfléchir ces deux phénomènes de manière distincte ; présentant à la fois des différences dans leurs formes et spécificités mais également dans les conséquences qu'ils entrainent respectivement [17].

Citons l'anonymat que procure Internet ainsi que la large diffusion qui peut être réalisée [7]. Notons également la permanence du cyber-harcèlement, qui suit l'adolescent tout au long de la journée et n'est donc plus restreint à l'espace scolaire ; ce dernier se trouvant dans l'impossibilité " d'échapper » à Internet en offrant à tous, à tous moments, la possibilité d'être informés et de visionner les commentaires, vidéos ou encore photos. Sourander et al. [15] ajoutent que le cyber-harcèlement permet une audience bien plus large que le harcèlement (qui se déroule généralement en petits comités) et que la victime éprouve davantage de difficultés à mobiliser les ressources pour stopper la situation de cyber-harcèlement ; augmentant l'impression de déséquilibre de pouvoir. Nous pouvons également observer que le cyber-harcèlement prive l'audience, y compris les victimes, d'indicateurs interpersonnels (ton de la voix, attitudes non-verbales...) qui renforcent le caractère subjectif de la situation, pouvant dès lors entrainer passivité au sein de l'audience et sentiment d'isolement chez la victime.

Quant aux conséquences engendrées, malgré la distinction entre ces deux formes de harcèlement, un consensus existe sur un lien fort entre l'implication (comme auteur ou victime) dans des actes de harcèlement classique et/ou de cyber-harcèlement et la présence de manifestations psychopathologiques chez l'adolescent [17]. La littérature s'accorde ainsi sur une cooccurrence entre ces deux formes, avec une victimisation traditionnelle s'associant à une cyber-victimisation et des conduites de harcèlement survenant avec celles du cyber-harcèlement [15]. Le harcèlement classique peut ainsi être perçu comme une prémices du cyber-harcèlement, au cours de laquelle l'auteur identifie une victime comme vulnérable et va étendre le harcèlement via la sphère numérique et virtuelle. En outre, des études [17] [e.g. 17] démontrent également que le fait d'être victime de harcèlement peut être associé au fait d'être auteur de cyber-harcèlement. L'anonymat des médias vir- tuels faciliterait ici le passage à l'acte de la victime par l'impression d'encourir moins de risques ; faisant ainsi évoluer un statut de victime à un statut de victime-auteur.

Malgré l'essor scientifique autour de ces phénomènes, et les distinctions qui existent concernant les modes d'apparition et de propagation, harcèlement et cyber-harcèlement semblent coexister à bien des égards. Les questions qui se posent sont dès lors de savoir si l'un découle de l'autre et si le cyber-harcèlement est notamment une nouvelle forme de harcèlement, ou si une existence isolée est (encore) possible dans une société où la réalité virtuelle prend de plus en plus de place, ce y compris dans les rapports sociaux. Il nous semble aujourd'hui nécessaire d'étudier ces phénomènes dans leurs interrelations et de les examiner conjointement quand on s'intéresse aux conduites de harcèlement au sens large chez l'adolescent, que ce soit à un niveau clinique ou empirique.

\section{L'empathie}

Plusieurs études se sont attachées à analyser les caractéristiques sociodémographiques concernant la santé mentale des adolescents ainsi que les facteurs de risque liés au harcèlement et cyberharcèlement. Toutefois, la clinique démontre également qu'il n'y a pas un profil unique qui caractérise les jeunes auteurs de harcèlement et/ou de cyber-harcèlement. Au contraire, divers facteurs psychologiques, sociaux et biologiques doivent être pris en compte dans la recherche de profils qui peuvent être utilisés pour identifier les adolescents à risque ou engagés dans ce type de conduites. Dans la section qui suit, nous nous attacherons à développer une composante essentielle qu'est l'empathie, capacité nécessaire au bon vivre ensemble et qui peut s'exprimer à différents niveaux.

Nous pouvons observer une corrélation forte entre les conduites antisociales et un faible niveau d'empathie au sein de la littérature scientifique, particulièrement concernant les situations de violence interpersonnelles [18], dont le harcèlement.

Avant de discuter des implications de l'empathie dans les conduites de harcèlement et de cyber-harcèlement, définissons au préalable ce qu'est l'empathie. L'empathie est une composante fondamentale de la vie sociale et émotionnelle d'un individu, participant tant à s'attacher à quelqu'un, qu'à comprendre ses émotions et celles d'autrui, à les interpréter et y réagir [19]. Plus précisément, l'empathie se décline principalement sous deux versants, l'empathie cognitive et l'empathie affective. La première fait référence à la capacité d'une personne de comprendre ses émotions et celles d'autrui ou plus globalement de prise de perspective émotionnelle, tandis que la seconde correspond à l'expression de l'empathie envers soi et les autres ou plus globalement de processus de contagion émotionnelle [19,20].

Concernant le harcèlement traditionnel, il est intéressant d'observer que l'association entre l'empathie, sous ces deux versants, et le fait de harceler n'est pas linéaire, principalement concernant l'empathie cognitive. En effet, certaines études [21] [e.g., 21] indiquent une association négative entre l'empathie cognitive et le harcèlement (c'est-à-dire que les adolescents capables de reconnaître et comprendre leurs émotions et celles des autres sont moins souvent auteurs de harcèlement et inversement) alors que d'autres n'indiquent pas d'association ou une association positive (c'est-à-dire que les adolescents capables de reconnaître et comprendre leurs émotions et celles des autres sont plus souvent auteurs de harcèlement ou inversement) [22]. Ce dernier résultat est plutôt interpellant et vient déconstruire l'idée que l'empathie est avant tout associée à des comportements pro sociaux. En effet, la fonction cognitive de l'empathie tient de la reconnaissance et de la compréhension mais pas forcément du partage [23]. Ajoutons à cela que cette fonction peut être assurée à un niveau personnel, où l'adolescent se montrerait plus attentif à ses propres émotions, 
et par là-même besoins, qu'à ceux perçus chez autrui ; ceci étant soutenu par le développement socio-affectif de l'adolescent, plus attaché aux bénéfices personnels immédiats. En outre, les situations de harcèlement sont hautement valorisables pour l'auteur sur le plan social, participant à sa popularité [20] et dès lors, augmentant les bénéfices perçus et pouvant court-circuiter l'anticipation ou le ressenti de culpabilité face au passage à l'acte [25].

L'empathie affective, elle, serait distinctement associée négativement au harcèlement et concorderait pour toutes ses formes particulières (physique, verbal, etc.) [18]. Aussi, la présence de niveaux élevés d'empathie affective permettrait de limiter les conduites de harcèlement en augmentant les possibilités de partages et d'échanges, en se mettant " à la place de "; empathie et expériences sociales s'influençant mutuellement.

Enfin, le cyber-harcèlement présenterait des liens plus constants pour les deux versants de l'empathie. En effet, le cyberharcèlement est associé à des niveaux plus faibles d'empathie à la fois cognitive et affective chez l'auteur [23]. L'absence voire l'ambiguïté de marqueurs non-verbaux, couplée aux interactions se réalisant virtuellement, à distance et dans l'immédiateté, pourraient être des éléments d'explications ; incitant davantage au repli sur soi, tant de ses affects que de ceux d'autrui, diminuant ainsi le seuil d'empathie par boucles rétroactives. D'autres recherches démontrent que l'empathie affective influencerait indirectement l'intention comportementale de cyber-harceler, au travers de variables socio-cognitives ; laissant à penser que des niveaux peu élevés d'empathie encourageraient le cyber-harcèlement via son efficacité perçue pour résoudre une situation interpersonnelle et via l'attribution de caractéristiques positives aux auteurs de cyberharcèlement (perçus comme cool, populaire...) [26].

En conclusion, il semblerait que l'idée d'un harceleur dénué d'empathie, voire de culpabilité, même si ces deux concepts sont distincts [25], soit peu vérifiée par la littérature scientifique. Aussi, nous sommes plutôt face à des auteurs de harcèlement qui agissent par profit personnel, sans pour autant être insensibles à autrui, proches du profil d'agresseur proactif [27] ou plus globalement ne présentant pas de profil antisocial avéré. La littérature observe par ailleurs que la composante sadique se retrouve peu dans le profil du harceleur, couplée à la période instable de l'adolescence, non propice à la cristallisation d'une structure de personnalité psychopathique [28]. Ceci est plutôt rassurant et ouvre des perspectives quant aux actions à développer.

\section{Pour une action intégrée}

Il nous semble nécessaire de replacer la conduite de harcèlement au sein d'un écosystème et d'intervenir à chacun des niveaux afin de prendre en charge efficacement ce phénomène, incluant l'ensemble des protagonistes. Nous aborderons plusieurs pistes, d'inspiration largement cognitivo-comportementale, provenant du harcèlement traditionnel pour ensuite conclure par des actions spécifiques concernant les situations de cyber-harcèlement, en adéquation avec le positionnement théorique développé précédemment.

Premièrement, il nous semble vain et même contre-productif d'axer la réponse au harcèlement sur les plans individuels et particulièrement, sur une confrontation entre l'auteur et sa victime, par quel que professionnel que ce soit, du moins comme réaction principale [14]. En effet, cette situation apprendrait d'une part que la victime ne peut se défendre seule, ce qui renforcerait le déséquilibre de pouvoir perçu, et accroitrait par la même occasion la visibilité de l'auteur, d'autant plus si l'action mise en place ne permet pas un arrêt de la situation de harcèlement. Comme discuté, l'auteur de harcèlement ne présente pas forcément un déficit d'empathie qu'il s'agirait de " corriger ", en mettant ce dernier face aux conséquences directes de ses actes, c'est-à-dire en présence de sa victime. Concernant celle-ci, les excuses entendues, si elles sont exprimées, résonneraient peu si elles ne sont pas suivies d'actes signalant la fin des brimades vécues. Ce type d'action présente donc, à notre sens, un enjeu davantage communautaire, signifiant que ce type de conduites est interdit et puni au sein de l'établissement scolaire, voire constitue une infraction au code pénal ; réaction qui peut être immédiate et ne nécessite pas la présence physique de la victime. Précisons toutefois, que, selon les situations, la mise en place d'une médiation et action de restauration envers la victime peut avoir du sens, ce dans un second temps. À cet égard, il s'avère nécessaire, pour une compréhension et un respect de la loi, que la terminologie juridique concernant le phénomène du harcèlement, mais plus encore du cyber-harcèlement, se précise afin de correspondre à la pluralité des situations rencontrées (e.g., reconnaissance et catégorisation de l'agressivité relationnelle dans le code pénal et conséquences associées) et des protagonistes impliqués (quid des témoins actifs, rôle de l'établissement scolaire...).

Dans le cadre de cet article, nous proposons d'intégrer dans la prise en charge l'une des fonction du harcèlement pour l'auteur, qui consiste à cultiver sa popularité et maintenir son statut social au sein de son groupe de pairs [29]. En effet, un des défis majeurs de l'adolescence consiste à développer des liens sociaux en dehors de la sphère familiale, participant au développement d'une intimité affective. Concernant les auteurs de harcèlement, il importerait de valoriser auprès d'eux d'autres compétences qui sont associées à la dominance sociale (être physiquement athlétique, être persuasif, confier des tâches/responsabiliser...) [24] ; contribuant à reconnaître et travailler cette fonction du harcèlement plutôt que la supprimer.

Afin de développer l'empathie, dont affective, des valeurs telles que la coopération doivent s'incarner au quotidien. La recherche, dont une méta-analyse sur le sujet assortie de recommandations pour les milieux de terrain, démontre que la cible privilégiée des actions à l'égard du harcèlement sont le groupe de pairs et les témoins de ces situations [1,24]. Plusieurs rôles ont été identifiés, tels que les soutiens à l'auteur, les défenseurs de la victime ou encore les témoins (passifs), ceux qui par leur simple présence renforcent positivement la situation de harcèlement en augmentant l'aura de l'auteur et justifiant son acte [30]. En parallèle, développer le sentiment d'auto-efficacité ${ }^{2}$ de la victime à gérer la situation de harcèlement (que ce soit via le soutien reçu directement et/ou une riposte verbale menaçant le statut social du harceleur) permet à celle-ci d'augmenter son sentiment de contrôle sur la situation et de dépasser un statut victimaire passif. Cela peut aussi contribuer à diminuer le sentiment de toute puissance de l'auteur et apprendre aux témoins que les statuts sociaux sont dynamiques et mouvants [31]. Aussi, il importe d'agir sur les témoins des situations de harcèlement, en développant davantage le soutien et l'empathie à l'égard des victimes d'une part et d'autre part en travaillant sur les normes sociales $^{3}$ et perceptions personnelles ${ }^{4}$ à l'encontre du harcèlement

\footnotetext{
2 Le sentiment d'auto-efficacité correspond à la croyance d'un individu en sa capacité de réaliser une tâche ou performance particulière. Bandura, A. The explanatory and predictive scope of self-efficacy theory. Journal of social and clinical psychology. 1986;4(3):359-73.

3 Dans ce cadre, les normes sociales sont communément décrites comme les perceptions d'un individu des prévalences et incidences d'une conduite donnée ici le harcèlement, au sein de son environnement proximal (école, classe, groupe d'amis...) (normes descriptives). Cela inclut également la façon dont l'individu perçoit que son environnement approuve cette conduite (normes subjectives). Rivis A., \& Sheeran, P. Social influences and the theory of planned behaviour: Evidence for a direct relationship between prototypes and young people's exercise behaviour. Psychology and Health. 2003;18(1):567-83.

4 Dans ce cadre, les perceptions personnelles correspondent à la perception d'un individu d'une conduite donnée, ici le harcèlement, en tant que immoral/moral blessant/pas blessant, etc. D'autres variables socio-cognitives peuvent être
} 
ou plus généralement de l'agressivité instrumentale, qui implique une tolérance zéro chez les élèves. Des interventions probantes existent au niveau européen [32], des expérimentations prometteuses se développent aux USA [24] et plus localement, des actions innovantes commencent à s'implanter ${ }^{5}$.

En outre, il est nécessaire que ces valeurs de coopération et de réactivité quant aux situations de harcèlement s'incarnent également au sein des classes, dans les lieux communs et activités des établissements scolaires. En effet, le harcèlement est peu présent dans les écoles avec un projet pédagogique cohérent, associé à des niveaux peu élevés de conflits. Ces écoles se centrent prioritairement sur le bien-être de l'élève et le développement de ses compétences émotionnelles et relationnelles, en parallèle de celles plus cognitives. La coopération prend le pas sur la compétition au travers de tâches et réalisations d'élèves, qui développent la diversité, le partage d'idées et la construction de projets communs plutôt qu'un modèle d'excellence individualiste.

Concernant le cyber-harcèlement, outre ce qui vient d'être développé, la composante virtuelle, spécificité du cyber-harcèlement, se doit d'être intégrée dans les actions à mettre en place. Ainsi, l'utilisation de vidéos illustrant des situations de cyberharcèlement et visant à développer l'empathie pour la victime, tout comme questionnant les normes sociales et perceptions personnelles au sein du groupe de pairs s'avéreraient intéressant comme première étape. Couplé ensuite à la présence de pairs formés à modérer les échanges sur les réseaux sociaux sur une période déterminée [33]. Ce type de dispositif intègre une gestion autonome des adolescents mais supervisée par l'adulte. En outre, l'encadrement des parents concernant l'usage d'internet et des réseaux sociaux est primordiale et se joue à deux niveaux. D'une part, un contrôle et une limitation du temps passé sur internet, associés à un rappel des règles de confidentialité des données personnelles [34], et d'autre part, une attitude d'ouverture et de discussion quant aux contenus visionnés et aux messages reçus, davantage en lien avec les dimensions de sollicitation et de confidences de la supervision parentale [35]. Enfin, le rapport de tout un chacun avec les technologies de l'information et de la communication est à questionner, notamment comme outil d'expression narcissique, ce en écho des modèles offerts aux jeunes.

\section{Conclusion}

Il serait tentant de parvenir à l'identification de profils clairs concernant les auteurs et les victimes, voire le double statut également rencontré, et de restreindre notre compréhension du harcèlement et du cyber-harcèlement à ces protagonistes. Il pourrait aussi être souhaitable de pointer la responsabilité unique de l'auteur et le viser dans l'action à mettre en place. Toutefois, les données scientifiques ne vont pas dans ce sens. Cet article a dès lors tenté de préciser les phénomènes de harcèlement et de cyber-harcèlement, d'en discuter les similarités et d'en délimiter les frontières, de discuter de la place de l'empathie et enfin de proposer des pistes d'action pratiques, de nature cognitivocomportementale. Même si nous envisageons une action intégrée, des prises en charges individuelles ne sont évidemment pas

mentionnées telles que le sentiment d'efficacité perçu de recourir à une telle conduite, les attentes de résultats, ou encore l'attribution de caractéristiques perçues chez l'auteur. Rivis, A., \& Sheeran, P. Social influences and the theory of planned behaviour: Evidence for a direct relationship between prototypes and young people's exercise behaviour. Psychology and Health. 2003;18(1):567-83.

5 https://www.reseau-prevention-harcelement.be/products/exemples-de-deuxmethodes-d-intervention-centrees-sur-la-victime-en-cas-de-situation-de-harcele ment/, https://www.preoccupationpartagee.org/decouvrir-la-methode/. Le lecteur peut consulter ces différentes sources pour avoir des informations plus précises sur les dispositifs d'action mentionnés. exclues, selon les singularités de l'auteur et les besoins de la victime. À l'instar de Malti [25], il nous semblait intéressant de se pencher davantage sur des actions cherchant à susciter des émotions morales positives chez chacun des protagonistes, telles que l'empathie (ou encore la fierté ou l'honneur), plutôt que de pointer l'absence de pensées morales ou la présence de pensées morales négatives (telles que la culpabilité ou la honte) (voir les travaux de Wikström) [36].

\section{Déclaration de liens d'intérêts}

Les auteurs déclarent ne pas avoir de liens d'intérêts.

\section{Références}

[1] Bradshaw CP. Translating research to practice in bullying prevention. Am Psychol 2015;70(4):322-32.

[2] Modecki KL, Minchin J, Harbaugh AG, Guerra NG, Runions KC. Bullying prevalence across contexts: a meta-analysis measuring cyber and traditional bullying. J Adolesc Health 2014;55(5):602-11.

[3] Waasdorp TE, Pas ET, Zablotsky B, Bradshaw CP. Ten-year trends in bullying and related attitudes among 4th-to 12th-graders. Pediatrics 2017;139(1):2016-615.

[4] Tolmatcheff C, Hénoumont F, Klée E, Galand B. Stratégies et réactions des victimes et de leur entourage face au harcèlement scolaire : une étude rétrospective. Psychologie Française 2019;64:391-407.

[5] Machimbarrena JM, Garaigordobil M. Prevalence of bullying and cyberbullying in the last stage of primary education in the Basque Country. Span J Psychol 2018;21(1):1-10

[6] Bottino SMB, Bottino C, Regina CG, Correia AVL, Ribeiro WS. Cyberbullying and adolescent mental health: systematic review. Cad Saude Publica 2015;31:463-75.

[7] Tsitsika A, Janikian M, Wójcik S, Makaruk K, Tzavela E, Tzavara C, et al. Cyberbullying victimization prevalence and associations with internalizing and externalizing problems among adolescents in six European countries. Comput Hum Behav 2015;51(1):1-7.

[8] Baldry AC, Sorrentino A, Farrington DP. Cyberbullying and cybervictimization versus parental supervision, monitoring and control of adolescents' online activities. Child Youth Serv Rev 2019;96(1):302-7.

[9] Whittaker E, Kowalski RM. Cyberbullying via social media. J Sch Violence 2015;14(1):11-29.

[10] Olweus D. Bully/victim problems among schoolchildren: long-term consequences and an effective intervention program. In: Hodgins S, editor. Mental disorder and crime. Thousand Oaks, CA, US: Sage Publications; 1993. p. 317-49.

[11] Smith PK, Barrio CD, Tokunaga ARS. Definitions of bullying and cyberbullying: how useful are the terms? In: Bauman S, Walker J, Cross D, editors. Principles of cyberbullying research: definition, methods, and measures. New York \& London: Routledge; 2012. p. 64-86.

[12] Jimerson SR, Swearer SM, Espelage DL. Handbook of bullying in schools: an international perspective. New York: Routledge; 2009.

[13] Gladden RM, Vivolo-Kantor AM, Hamburger ME, Lumpkin CD. Bullying surveillance among youths, 1st ed National Center for Injury Prevention and Control, Centers for Disease Control and Prevention and the United States Department of Education; 2014.

[14] Ttofi MM, Farrington DP. Effectiveness of school-based programs to reduce bullying: a systematic and meta-analytic review. J Exp Criminol 2011;7(1):27-56.

[15] Sourander A, Klomek AB, Ikonen M, Lindroos J, Luntamo T, Koskelainen M, et al. Psychosocial risk factors associated with cyberbullying among adolescents: a population-based study. Arch Gen Psychiatry 2010;67(7):720-8.

[16] Rivers I, Poteat VP, Noret N, Ashurst N. Observing bullying at school: the mental health implications of witness status. Sch Psychol Q 2009;24(4):211-23.

[17] Arsène M, Raynaud J-P. Cyberbullying (ou cyber harcèlement) et psychopathologie de l'enfant et de l'adolescent: état actuel des connaissances. Neuropsychiatr Enfance Adolesc 2014;62(4):249-56.

[18] van Noorden THJ, Haselager GJT, Cillessen AHN, Bukowski WM. Empathy and involvement in bullying in children and adolescents: a systematic review. J Youth Adolesc 2015;44(3):637-57.

[19] Decety J, Cowell JM. The complex relation between morality and empathy. Trends Cogn Sci 2014;18(7):337-9.

[20] Vachon DD, Lynam DR. Fixing the problem with empathy: development and validation of the affective and cognitive measure of empathy. Assessment 2016;23(2):135-49.

[21] Fougeret-Linlaud V, Catheline N, Chabaud F, Gicquel L. Le harcèlement scolaire entre pairs. À propos d'une étude en Vienne visant à évaluer l'apport d'un support ludique mettant en jeu les émotions. Neuropsychiatr Enfance Adolesc 2016;64(4):216-23.

[22] Caravita SCS, Blasio PD, Salmivalli C. Unique and interactive effects of empathy and social status on involvement in bullying. Soc Dev 2009;18(1):140-63.

[23] Sticca F, Ruggieri S, Alsaker F, Perren S. Longitudinal risk factors for cyberbullying in adolescence: longitudinal risk factors for cyberbullying. J Community Appl Soc Psychol 2013;23(1):52-67. 
[24] Ellis BJ, Volk AA, Gonzalez J-M, Embry DD. The meaningful roles intervention: an evolutionary approach to reducing bullying and increasing prosocial behavior. J Res Adolesc 2016;26(4):622-37.

[25] Malti T. Toward an integrated clinical-developmental model of guilt. Dev Rev 2016;39:16-36

[26] Lazuras L, Barkoukis V, Ourda D, Tsorbatzoudis H. A process model of cyberbullying in adolescence. Comput Hum Behav 2013:29(3):881-7.

[27] Volk AA, Dane AV, Marini ZA. What is bullying? A theoretical redefinition. Dev Rev 2014;34(4):327-43.

[28] Book AS, Volk AA, Hosker A. Adolescent bullying and personality: an adaptive approach. Personal Individ Differ 2012;52(2):218-23.

[29] Dijkstra JK, Lindenberg S, Veenstra R. Beyond the class norm: bullying behavior of popular adolescents and its relation to peer acceptance and rejection. J Abnorm Child Psychol 2008;36(8):1289-99.

[30] Salmivalli C. Bullying and the peer group: a review. Aggress Violent Behav 2010;15(2):112-20.
[31] Piquet E. Je me défends du harcèlement. Paris: Albin Michel Jeunesse; 2016.

[32] Salmivalli C, Poskiparta E. KiVa antibullying program: overview of evaluation studies based on a randomized controlled trial and national rollout in Finland. Int J Confl Violence 2012;6(2):294-302.

[33] Palladino BE, Nocentini A, Menesini E. Evidence-based intervention against bullying and cyberbullying: evaluation of the NoTrap! program in two independent trials: evaluation of the NoTrap! program. Aggress Behav 2016;42(2):194-206.

[34] Cassidy W, Faucher C, Jackson M. Cyberbullying among youth: a comprehensive review of current international research and its implications and application to policy and practice. Sch Psychol Int 2013;34(6):575-612.

[35] Kerr M, Stattin H, Burk WJ. A reinterpretation of parental monitoring in longitudinal perspective. J Res Adolesc 2010;20(1):39-64.

[36] Wikström POH. Explaining crime as moral actions. In: Handbook of the sociology of morality. New-York: Springer; 2010. p. 211-39.

Pour citer cet article : Mathys C, Claes S. Conduites de harcèlement et de cyber-harcèlement chez les adolescents : interrelations et spécificités, place de l'empathie et actions de prise en charge. Neuropsychiatr Enfance Adolesc (2020), https://doi.org/10.1016/j.neurenf.2020.02.003 